[0212-7199 (2005) 22: 4; pp 177-181] ANALES DE MEDICINA INTERNA Copyright (C) 2005 ARAN EDICIONES, S.L

An. MED. INTERNA (Madrid) Vol. 22, N. ${ }^{\circ} 4$, pp. 177-181, 2005

\section{Tratamiento del tromboembolismo pulmonar agudo con heparina de bajo peso molecular subcutánea. Comparación con heparina no fracionada intravenosa}

\author{
J. L. ALONSO MARTÍNEZ, M. L. ABÍNZANO GUILLÉN, M. SOLANO REMÍREZ, \\ M. T. ÁLVAREZ FRÍAS, J. GUTIÉRREZ DUBOIS, L. MUNUERA GARCÍA
}

Servicio de Medicina Interna. Hospital de Navarra. Pamplona
LOW-MOLECULAR-WEIGHT HEPARIN FOR THE TREATMENT OF ACUTE PULMONARY THROMBOEMBOLISM. COMPARISON WITH \author{
UNFRACTIONATED INTRAVENOUS HEPARIN
}

\section{RESUMEN}

Fundamento y objetivos: Desde la introducción en la terapéutica de las heparinas de bajo peso molecular, éstas se han venido utilizando con una eficacia similar o superior a la heparina no fraccionada para el tratamiento de la enfermedad tromboembólica venosa. Nuestro propósito fue conocer la eficacia de enoxaparina en el tratamiento del tromboembolismo pulmonar agudo.

Métodos: Comparamos la eficacia de enoxaparina subcutánea dos veces al día a dosis de $1 \mathrm{mg} / \mathrm{kg}$ de peso con la de heparina no fraccionada por vía endovenosa de forma continua en pacientes diagnosticados de tromboembolismo pulmonar agudo determinando la tasa de hemorragia mayor, muerte en el episodio índice y tasa de reicidiva. Como tromboembolismo pulmonar masivo se consideró la visualización de trombos en las arterias pulmonares principales.

Resultados: Treinta y ocho pacientes fueron tratados con heparina no fraccionada intravenosa de forma continua (edad $72 \pm 9$ años, varón $58 \%$, tromboembolismo pulmonar masivo $24 \%$ ) y 65 pacientes fueron tratados con enoxaparina (edad $71 \pm 12$ años, varón 52\%, tromboembolismo pulmonar masivo $49 \%$ ).

La tasa de hemorragia mayor durante la hospitalización índice fue de $8 \%$ en el grupo de heparina no fraccionada y de $3 \%$ en el grupo de enoxaparina (riesgo relativo 5,2; diferencia de riesgos 0,63 ; reducción de episodios de $37 \%$ CI $95 \%-0,16$ a $0,06 \%, p=0,21$ ), la tasa de muerte intrahospitalaria fue de $8 \%$ en el grupo de heparina no fraccionada y de $1,5 \%$ en el grupo enoxaparina (riesgo relativo 1,52 ; diferencia de riesgos 1,54; reducción de muerte de $25 \%$, CI $95 \%-0,17$ a $0,04 \%, p=0,11)$. La tasa de recidiva fue de $44 \%$ en el grupo de tratados con heparina no fraccionada y de $13 \%$ en el grupo de enoxaparina (riesgo relativo 1,80; riesgo atribuible 6,48; reducción de riesgo de $30 \%$, CI $95 \%-0,60$ a $0,02, \mathrm{p}=$ $0,01)$

Conclusión: El tratamiento del tromboembolismo pulmonar agudo con heparina de bajo peso molecular (enoxaparina) es más eficaz que el tratamiento con heparina no fraccionada de forma continua, produciéndose menos hemorragias, menos muertes intrahospitalarias y menor tasa de recidivas, aun cuando el tromboembolismo pulmonar sea masivo.

PALABRAS CLAVE: Enfermedad tromboembólica venosa. Heparina de bajo peso molecular. Enoxaparina. Tratamiento.

\section{ABSTRACT}

Background and objectives: Low-molecular-weight heparins have been demonstrated at least as useful as unfractionated heparin (UFH) in the treatment of venous thromboembolic disease. Our aim was to know the effectivity and security of subcutaneous enoxaparin in the treatment of acute pulmonary embolism.

Methods: We compared the effectivity and security of two doses daily, subcutaneous injected enoxaparin adjusted to body weight, and standard treatment with continuous intravenous UFH, determining the rate of major bleeding, in-hospital death and recurrent venous thromboembolic disease in long-term follow up. Massive pulmonary thromboembolism was defined as thrombotic material seen in main pulmonary arteries.

Results: Thirty eight patients were treated with UFH (Mean age 72 SD 9 years, male 58\%, massive pulmonary thromboembolism 24\%) and 65 patients were treated with subcutaneous enoxaparin (Mean age 71 SD 12 years, male 52\%, massive pulmonary thromboembolism 49\%).

Major bleeding rate was $8 \%$ in UHF group and $3 \%$ in enoxaparin group (Difference 37\%, 95\% Confidence interval -0.16 to $0.06, p=0.21$ ). In-hospital death rate was $8 \%$ in UHF group and $1.5 \%$ in enoxaparin group (Difference 25\%, 95\% Confidence interval -0.17 to $0.04, p=0.11$ ). Recurrent thromboembolism rate was $44 \%$ in UFH group and $13 \%$ in enoxaparin group (Difference 30\%, 95\% Confidence interval -0.60 to $0.02, p=0.01$ )

Conclusion: Our findings demonstrate that treatment of acute pulmonary thromboembolism with low-molecular-heparin is effective and safe, even in massive pulmonary embolism.

KEY WORDS: Venous thromboembolic disease. Low molecular weight heparin. Enoxaparin. Treatment.

Alonso Martínez JL, Abínzano Guillén ML, Solano Remírez M, Álvarez Frías MT, Gutiérrez Dubois J, Munuera García L. Tratamiento del tromboembolismo pulmonar agudo con heparina de bajo peso molecular subcutánea. Comparación con heparina no fracionada intravenosa. An Med Interna (Madrid) 2005; 22: 177-181. 


\section{INTRODUCCIÓN}

La heparina ha demostrado ser imprescindible en el tratamiento de la fase aguda del tromboembolismo pulmonar, reduciendo la mortalidad y las recidivas de la enfermedad tromboembólica venosa (1-3). En los últimos años varios estudios han enfocado el tema de la efectividad de las heparinas de bajo peso molecular en el tratamiento de la enfermedad tromboembólica venosa, siendo al parecer tan eficaces como la heparina no fraccionada administrada por vía endovenosa de forma continua (4-8). Sin embargo los estudios han sido en su mayor parte numéricamente pequeños, con pocos estudios incluyendo más de 200 pacientes y en general incluyendo pacientes afectos de trombosis venosa aislada mezclados con pacientes afectos de la manifestación mas grave de la enfermedad tromboembólica venosa, como es el tromboembolismo pulmonar (9-13).

Varios metanálisis han tratado el problema concluyendo que el tratamiento con heparinas de bajo peso molecular del tromboembolismo pulmonar es igual o superior al tratamiento clásico con heparina no fraccionada administrada de forma continua por vía endovenosa. No obstante, al analizar pacientes mezclados con trombosis venosa profunda aislada la selección de trabajos y pacientes puede ser problemática. Además muchos de los pacientes analizados padecían tromboembolismo pulmonar submasivo, por lo que la interpretación de los resultados puede ser difícil (14-17).

Presentamos datos comparativos de dos grupos de pacientes con tromboembolismo pulmonar agudo sintomáticos tratados con heparina no fraccionada endovenosa continua un grupo y con heparina de bajo peso molecular el otro.

\section{MATERIAL Y MÉTODOS}

Comparamos la efectividad del tratamiento con heparina no fraccionada por vía intravenosa de forma continua con el tratamiento con heparina de bajo peso molecular (enoxaparina) por vía subcutánea en dos dosis de $1 \mathrm{mg} / \mathrm{kg}$ de peso en pacientes ingresados por tromboembolismo pulmonar agudo en nuestra área de hospitalización de medicina interna en un registro de 10 años, de 1992 a 2002: los primeros 5 años los pacientes fueron tratados con heparina no fraccionada por vía endovenosa de forma continua, y los últimos 5 años con heparina subcutánea de bajo peso molecular (18).

Observamos la mortalidad hospitalaria, la mortalidad en el seguimiento, la tasa de hemorragia mayor, la tasa de recidiva de la enfermedad tromboembólica venosa y la tasa de cáncer.

Se definió el tromboembolismo pulmonar agudo como la existencia de manifestaciones clínicas (combinaciones de disnea, dolor torácico, sincope, edemas y dolor en extremidades) junto con la demostración de material trombótico en arterias pulmonares por arteriografía pulmonar o tomografía computarizada helicoidal torácica o gammagrafía pulmonar de ventilación-perfusión de alta probabilidad más demostración de trombosis venosa por flebografía o ecografía doppler color. Consideramos tromboembolismo pulmonar masivo cuando se observó material trombótico en las arterias pulmonares principales, bien en el tronco común de la arteria pulmonar o en las arterias pulmonares principales, y no masivo cuando era periférico, bien lobar o segmentario.

Como hemorragia mayor se consideraron aquellas que ocurrieron durante el ingreso por el tromboembolismo pulmo- nar y que causaron descenso del hematocrito igual o mayor a $2 \%$, que precisaron transfusión de sangre o alargamiento del ingreso hospitalario.

Calculamos el gradiente alveolo-arterial para el oxígeno con la fórmula:

$\mathrm{A}-\mathrm{a} \mathrm{O}_{2}=\left[\mathrm{FiO}_{2}-(\mathrm{Pb}-47)-\left(\mathrm{PACO}_{2} / \mathrm{R}\right)\right]+\left[\mathrm{FiO}_{2}(1-\mathrm{R})\right.$ $\left(\mathrm{PACO}_{2} / \mathrm{R}\right]-\mathrm{PaO}_{2}$

Donde es $\mathrm{FiO}_{2}$ el flujo de oxígeno, $\mathrm{Pb}$ la presión barométrica, 47 la presión del vapor de agua, $\mathrm{PACO}_{2}$ es equivalente a la $\mathrm{PaCO}_{2}, \mathrm{R}$ es el cociente respiratorio, normalmente 0,8 y $\mathrm{PaO} 2$ es la presión arterial de oxígeno.

El dímero D se determinó por técnica ELISA y la gasometría arterial y la LDH según técnica habitual del laboratorio del hospital.

Análisis estadístico: Asumiendo una tasa de mortalidad hospitalaria del 2\% para el tromboembolismo pulmonar agudo, calculamos el tamaño de la muestra para un error alfa de 0,05 y una potencia del $80 \%$, estimando el número necesario de pacientes por grupo en 36. Calculamos el riesgo relativo y el riesgo atribuible utilizando como muestra de población los pacientes tratados clásicamente con heparina no fraccionada endovenosa durante el periodo de tiempo de 10 años.

Para comparación de variables continuas utilizamos la prueba de la t de Student y la u de Mann Whitney para variables no paramétricas. Para comparar variables discretas utilizamos la comparación de proporciones y la prueba exacta de Fisher. Utilizamos la prueba de la Q de Yule para evaluar la fuerza de asociación entre variables discretas. Consideramos significación estadística valores de $\mathrm{p}<0,05$.

\section{RESULTADOS}

En la Tabla I se muestran los datos referentes a edad y sexo de los pacientes, observando que no existen diferencias entre ambos grupos.

Del mismo modo, en la Tabla I se reflejan datos de tensión arterial sistólica, gasométricos, días de retraso en el diagnostico (referidos como días de clínica previos al diagnóstico y tratamiento), nivel de LDH y dímero D, apreciándose que los dos grupos son homogéneos en cuanto a gravedad del episodio de tromboembolismo pulmonar.

La proporción de tromboembolismo afectando a arterias pulmonares principales, pudiéndose considerar como tromboembolismo masivo, fue mayor en el grupo de pacientes tratados con heparina de bajo peso molecular, sin embargo este hecho no influyó en la tasa de mortalidad.

En la Tabla II se muestran la tasa de hemorragia durante el episodio índice. Ocurrieron 5 episodios de hemorragia clínicamente mayor: Dos hemorragias digestivas altas, 1 hemorragia digestiva baja y 2 hematomas musculares. La proporción de hemorragias fue similar pata ambos grupos de tratamiento.

La tasa de mortalidad en ambos grupos (Tabla II), tanto en el episodio de hospitalización como en el seguimiento, fue similar entre ambos grupos. Así mismo, tampoco hubo diferencias en la tasa de cáncer entre ambos grupos, tanto la de los pacientes ya diagnosticados de cáncer como los nuevos diagnósticos en el seguimiento. 
TABLA I

COMPARACIÓN DE EDAD, SEXO Y DATOS CLÍNICOS EN PACIENTES TRATADOS CON HEPARINA NO FRACCIONADA CONTINUA Y ENOXAPARINA

\begin{tabular}{|c|c|c|c|c|}
\hline & HBPM & HNF & IC $95 \%$ & $p$ \\
\hline$n$ & 65 & 38 & & \\
\hline Edad & $71 \pm 12$ & $72 \pm 9$ & & NS \\
\hline $\operatorname{Sexo}(M / F)$ & $34 / 31$ & $22 / 16$ & $-0,15$ a 0,28 & NS \\
\hline TAS & $119 \pm 16$ & $126 \pm 25$ & & NS \\
\hline Pulso & $87,78 \pm 15,29$ & $91,55 \pm 14,22$ & & NS \\
\hline Temperatura & $36,9 \pm 0,75$ & $36,9 \pm 0,69$ & & NS \\
\hline $\mathrm{pH}$ & $7,41 \pm 0,26$ & $7,44 \pm 0,04$ & & NS \\
\hline $\mathrm{PaO}_{2}$ & $60,27 \pm 14,10$ & $62,22 \pm 12,17$ & & NS \\
\hline $\mathrm{PaCO}_{2}$ & $32,33 \pm 5,54$ & $33,03 \pm 4,69$ & & NS \\
\hline $\mathrm{A}-\mathrm{aO}_{2}$ & $49,87 \pm 11,86$ & $47,04 \pm 14,81$ & & NS \\
\hline $\mathrm{LDH}^{2}$ & $265 \pm 105$ & $251 \pm 87$ & & NS \\
\hline Días de clínica & $10,12 \pm 12,51$ & $9,72 \pm 12,67$ & & 0,07 \\
\hline Dimero D & $5,1 \pm 6,17$ & $3,65 \pm 5,32$ & & 0,06 \\
\hline TEP central & $29(49 \%)$ & $9(24 \%)$ & $-0,40$ a 0,027 & $=0,02$ \\
\hline
\end{tabular}

HBPM: heparina de bajo peso molecular (enoxaparina); HNF: heparina no fraccionada intravenosa continua; $\mathrm{Pa}_{2}$ : Presión parcial de oxígeno en sangre arterial; $\mathrm{PaCO}_{2}$ : Presión parcial de dioxido carbónico en sangre arterial; $\mathrm{A}-\mathrm{aO}_{2}$ : Gradiente alveolo-arterial de oxígeno; TEP: tromboembolismo pulmonar; NS: no significación estadística.

\section{TABLA II}

\begin{tabular}{|c|c|c|c|c|}
\hline \multicolumn{5}{|c|}{$\begin{array}{l}\text { COMPARACIÓN DE TASAS DE HEMORRAGIA, MUERTE, } \\
\text { CÁNCER Y RECIDIVA }\end{array}$} \\
\hline & HBPM & HNF & IC $95 \%$ & $p$ \\
\hline Hemorragia & $2(3 \%)$ & $3(8 \%)$ & $-0,16$ a 0,06 & 0,21 \\
\hline Exitus (hospitalización) & $1(1,5 \%)$ & $3(8 \%)$ & $-0,17$ a 0,04 & 0,11 \\
\hline Exitus (seguimiento) & $19(29 \%)$ & $17(45 \%)$ & $-0,36$ a 0,05 & NS \\
\hline Cáncer total & $21(32 \%)$ & $10(26 \%)$ & $-0,14$ a 0,26 & NS \\
\hline Cáncer nuevo & $4(6 \%)$ & $5(13 \%)$ & $-0,21$ a 0,07 & NS \\
\hline Recidiva & $6(9 \%)$ & $8(21 \%)$ & $-0,28$ a 0,05 & NS \\
\hline Recidiva (supervivientes) & $6 / 45(13 \%)$ & $8 / 18(44 \%)$ & $-0,60$ a $-0,02$ & $=0,01$ \\
\hline Tiempo de recidiva & $6 \pm 3,3$ & $35 \pm 26,4$ & & $<0,05$ \\
\hline
\end{tabular}

HBPM: heparina de bajo peso molecular (enoxaparina); HNF: heparina no fraccionada intravenosa continua; NS: No significación estadística.

La tasa de recidiva fue significativamente mayor en los pacientes tratados con heparina sódica no fraccionada por vía endovenosa que en los tratados con enoxaparina. En el grupo tratado con heparina de bajo peso molecular la recidiva ocurrió como nuevo episodio de tromboembolismo en $5(11 \%)$ pacientes y como trombosis venosa profunda aislada en un paciente. En el grupo de pacientes tratados con heparina no fraccionada por vía endovenosa de forma continua fue de 7 $(39 \%)(\mathrm{p}=0,02)$ como nuevo tromboembolismo pulmonar y 1 como trombosis venosa profunda aislada.

Sin embargo, el tiempo de recidiva fue más corto en los pacientes tratados con heparina de bajo peso molecular si bien esta circunstancia hay que analizarla en relación con el tiempo de profilaxis secundaria y el tipo de anticoagulación: de los pacientes supervivientes realizaron tratamiento con cumarínicos $78(79 \%), 29(83 \%)$ de los pacientes del grupo de hepari-
TABLA III

RIESGO RELATIVO Y ATRIBUIBLE

\begin{tabular}{lccccc} 
& HNF & HBPM & RR & RA & Q \\
\hline Tasa de muerte inicial & 0,78 & 0,15 & 5,2 & 0,63 & 0,69 \\
Tasa de muerte (seguimiento) & 4,47 & 2,93 & 1,52 & 1,54 & $-0,20$ \\
Tasa de hemorragia & 0,78 & 0,30 & 2,6 & 0,48 & $-0,66$ \\
\hline
\end{tabular}

HBPM: heparina de bajo peso molecular (enoxaparina); HNF: heparina no fraccionada intravenosa continua; $\mathrm{RR}$ : riesgo relativo; $\mathrm{RA}$ : riesgo atribuible; Q: valor de la Q de Yule.

na no fraccionada y $47(73 \%)$ de los pacientes de grupo enoxaparina durante una media de $8 \pm 3,45$ meses y 21 pacientes siguieron profilaxis secundaria con diferentes heparinas de bajo peso molecular $6(7 \%)$ del grupo de heparina no fraccionada y $16(17 \%)$ del grupo enoxaparina, durante $5 \pm 3,6$ meses $(\mathrm{p}<0,001)$.

La tasa de muerte durante la hospitalización (Fig. 1) fue de 0,78 en el grupo de los tratados con heparina no fraccionada y de 0,15 en el grupo de heparina de bajo peso molecular (riesgo relativo 5,2 , riesgo atribuible 0,63 ). La tasa de muerte durante el seguimiento fue de 4,47 en el grupo de pacientes tratados con heparina no fraccionada y de 2,93 en el grupo de pacientes tratados con heparina de bajo peso molecular (riesgo relativo 1,52, riesgo atribuible 1,54). La tasa de hemorragia durante hospitalización fue de 0,78 en el grupo de pacientes tratados con heparina no fraccionada y de 0,30 en el grupo de pacientes tratados con heparina de bajo peso molecular (riesgo relativo 2,6 , riesgo atribuible 0,48 ).

La reducción de hemorragia fue de $37 \%$, de muerte del $25 \%$ y de recidiva del $30 \%$ en el grupo de enoxaparina respecto al grupo de heparina no fraccionada (Fig. 2).

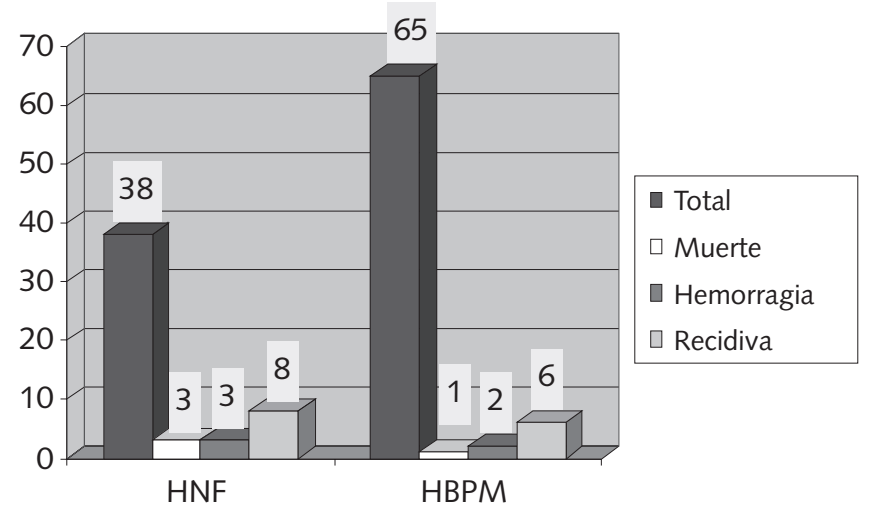

Fig. 1. Representación de frecuencias de hemorragia, muerte y recidiva (HNF: heparina no fraccionada; HBPM: heparina de bajo peso molecular).

\section{DISCUSIÓN}

Numerosos estudios prospectivos y aleatorizados han demostrado que el tratamiento de la enfermedad tromboem- 


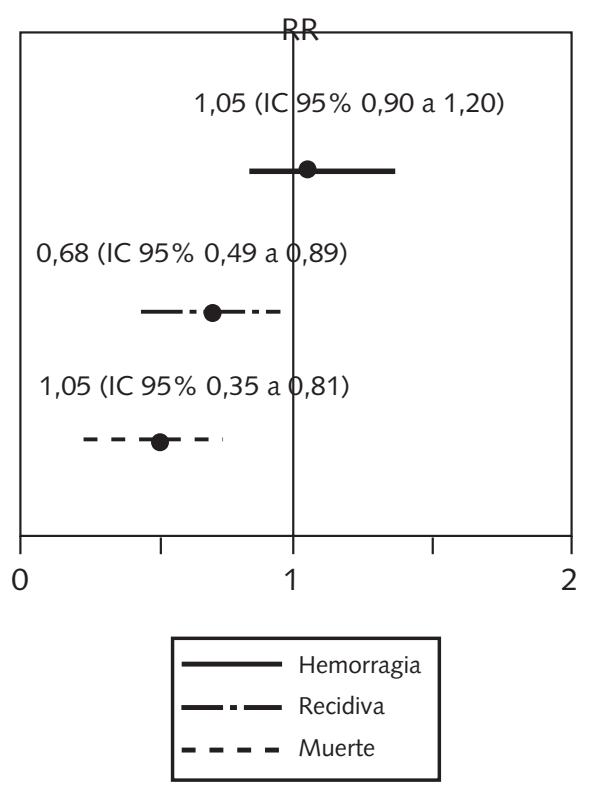

Fig. 2. Representación de disminución de riesgos (riesgo grupo $H B P M /$ riesgo grupo HNF. HNF: heparina no fraccionada; HBPM: heparina de bajo peso molecular).

bólica venosa con dosis fijas, ajustadas por peso, de diferentes heparinas de bajo peso molecular son al menos tan eficaces como el tratamiento clásico con heparina no fraccionada por vía endovenosa de forma continua, pero la mayoría de estos estudios han sido realizados en pacientes con trombosis venosa profunda (4-7), y pocos de ellos han sido llevados a cabo en pacientes con la forma más grave del espectro de la enfermedad tromboembólica venosa, el tromboembolismo pulmonar (8-11). En los estudios se han mezclado pacientes con trombosis venosa profunda y con tromboembolismo pulmonar, y pocos estudios son numéricamente importantes.

Los trabajos que tratan con heparina de bajo peso molecular ajustado por peso pacientes con tromboembolismo pulmonar, son en general tromboembolismos pulmonares periféricos no masivos $(12,13)$, y muchas de las conclusiones son extraídas de metanálisis, con el riesgo genérico del metanálisis de poder incrementar los resultados.

En nuestro trabajo, realizado a partir de un registro de 10 años de tromboembolismo pulmonar, tratamos los 5 primeros años con heparina no fraccionada de forma continua y con enoxaparina los siguientes 5 años (18). Vemos en los resultados que tanto la mortalidad durante la hospitalización por el episodio agudo, como la tasa de hemorragia mayor, como la tasa de recidivas posteriores de la enfermedad tromboembólica venosa son menores en los pacientes tratados con enoxaparina que en los tratados con heparina no fraccionada, con una reducción importante de eventos de entre el 25 y el $35 \%$ para los tres objetivos estudiados.

Además, en nuestra opinión, el tratamiento del tromboem- bolismo pulmonar con heparinas de bajo peso molecular ha significado una simplificación del tratamiento, permitiendo prescindir de bombas de perfusión, controles de coagulación y material fungible, junto con un aumento de la eficiencia en el tratamiento.

Factores asociados a la recurrencia de la enfermedad tromboembólica venosa son cuando se manifiesta como tromboembolismo pulmonar (19) así como en pacientes con cáncer, una duración corta de la prevención secundaria y en pacientes con trombosis venosa proximal (20).

Un dato que merece la pena comentario es que el tiempo de recidiva fue menor en los pacientes tratados con enoxaparina frente a los tratados con heparina no fraccionada. Existen datos en la literatura que reflejan una mayor tasa de recidiva de la enfermedad tromboembólica cuando existe un retraso en alcanzar un nivel de anticoagulación adecuado (21), es decir prolongar el tiempo parcial de tromboplastina activado hasta 1.5-2,5 veces el tiempo basal, cuando se usa en el tratamiento heparina no fraccionada, aunque los estudios en este sentido no hayan sido uniformes $(22,23)$. Usualmente en clínica el nivel de anticoagulación alcanzado con las heparinas de bajo peso molecular no se mide, por lo tanto la velocidad en alcanzar anticoagulación eficaz es desconocida, y podría alcanzarse anticoagulación más rápida inyectando un bolus de $5.000 \mathrm{U}$ de heparina sódica endovenosa a la vez que la dosis de heparina de bajo peso molecular subcutánea. Aunque el tiempo de recidiva haya sido más corto en los tratados con enoxaparina, en nuestro pacientes parece depender mas del tiempo de profilaxis secundaria que del tratamiento inicial, estando en consonancia con trabajos recientes que demuestran que la prolongación del tiempo de anticoagulación se asocia a una tasa menor de recidiva con un riesgo de hemorragia aceptable (24-26).

Las principales limitaciones del presente trabajo consisten en que la aleatorización no ha sido posible, se han tratado la mitad del tiempo con heparina no fraccionada y la otra mitad con enoxaparina. No obstante al no existir diferencias en la edad, parámetros hemodinámicos y gasométricos hace que los grupos puedan ser comparables. Además los pacientes con tromboembolismo pulmonar masivo eran más numerosos en el grupo de enoxaparina, con lo que la oportunidad de ir mal era mayor en este grupo, no habiéndose demostrado. Aun más, según estos datos los pacientes con tromboembolismo pulmonar masivo pueden ser eficientemente tratados con heparina de bajo peso molecular por vía subcutánea, aunque la definición de tromboembolismo pulmonar masivo aun está por definir claramente en la literatura: si es expresión del tamaño del vaso ocluido, del grado de inestabilidad hemodinámica, del grado de insuficiencia respiratoria o de los tres en conjunto.

Otra limitación importante de nuestro trabajo es el número de pacientes pequeño, del que adolecen la mayoría de los trabajos en la literatura. Sin embargo, aunque no puedan generalizarse las conclusiones, si puede ser útil como comunicación de nuestra experiencia en este campo y como parte de futuros análisis conjuntos con otras experiencias. 


\section{Bibliografía}

1. Barril DW, Jordan SJ. Anticoagulants drugs in the treatment of pulmonary embolism: A controlled trial. Lancet 1960; 1: 1309-12.

2. Brandjes DPM, Heijboer H, Büller HL, de Rijk M, Jagt H, ten Cate JW. Acenocoumarol and heparin compared with acenocoumarol alone in the initial treatment of proximal-vein thrombosis. N Engl J Med 1992; 327: 1485-89.

3. Hull RD, Raskob GE, Brant RF, Pineo GF, Valentine KA. The importance of initial treatment on long-term clinical outcomes of antithrombotic therapy. Arch Intern Med 1997; 157: 2317-21.

4. Prandoni P, Lensing AW, Buller HR, Carta M, Cogo A, Vigo M, et al Comparison of subcutaneous low-molecular-weight heparin with intravenous standard heparin in proximal deep-venous thrombosis. Lancet 1992; 339: 441-5.

5. Harenberg J, Schmidt JA, Koppenhagen K. Tolle A, Huisman MV, Buller HR. Fixed-dose, body weight-independent subcutaneous LMW heparin versus adjusted dose unfractionated intravenous heparin in the initial treatment of proximal venous-thrombosis. Eastern investigators. Thromb Haemost 2000; 83: 652-6.

6. The Columbus investigators. Low-molecular-weight heparin in the tratment of patients with venous thromboembolism. N Engl J Med 1997; 337-657-62.

7. Breddin HK, Hoch-Wunderle V, Nakov R, Kakkar VV. Effects of a low molecular weight heparin on thrombus regression and recurrent thromboembolism in patients with deep-vein thrombosis. N Engl J Med 2001; 344: 626-31.

8. Writting Committee for the Galilei Investigators. Subcutaneous adjusted-dose unfractionated heparin vs fixed-dose low-molecular-weight heparin in the initial treatment of venous thromboembolism. Arch Intern Med 2004; 164: 1077-83.

9. Simonneau G, Sors H, Charbonnier B, Page Y, Laaban JP, Azorian R, et al. A comparison of low-molecular-weight heparin with unfractionated heparin for acute pulmonary embolism. N Engl J Med 1997; 337: 663-9.

10. Merli G, Spiro TE, Olsson CG, Abildgaard U, Davidson BL, Eldor A, et al. Subcutaneous enoxaparin once or twice daily compared with intravenous unfractionated heparin for treatment of venous thromboembolic disease. Ann Intern Med 2001; 134: 191-202.

11. Beckman JA, Dunn K, Sasahara AA, Goldhaber SZ. Enoxaparin monotherapy without oral anticoagulation to treat acute symptomatic pulmonary embolism. Thromb Haemost 2003; 89: 952-8.

12. Thery C, Simonneau G, Meyer G, Helenon O, Bridey F, Armagnac C, et al. Randomized trial of subcutaneous low-molecular-weight heparin CY216 (Fraxiparine) compared with intravenous unfractionated heparin in the curative treatment of submassive pulmonary embolism. Circulation 1992; 85: 1380-9.

13. Meyer G, Brenot F, Pacouret G, Simonneau G, Gillet-Juvin K, Charbonnier B, et al. Subcutaneous low-molecular-weight-heparin fragmin versus intravenous unfractionated heparin in the treatment of acute non massive pulmonary embolism: An open randomized pilot study. Thromb Haemost 1995; 1432-5.
14. Hommes DW, Bura A, Mazzolai L, Buller HR, ten Cate JW. Subcutaneous heparin compared with continuous intravenous heparin administration in the initial treatment of deep-venous thrombosis: A metaanalysis. Ann Intern Med 1992; 116: 279-84.

15. Dalorich LR, Ginsberg JS, Doukets JD, Holbrook AM, Cheah G. A meta-analysis comparing low-molecular-weight heparins with unfractionated heparin in the treatment of venous thromboembolism: examining some unanswered questions regarding location of treatment, product type and dose frequency. Arch Intern Med 2000; 160: 181-8.

16. Gould MK, Dembitzer AD, Doyle RL, Hastie TJ, Garber AM. Lowmolecular-weight heparin compared with intravenous unfractionated heparin for treatment of acute deep venous thrombosis. A meta-analysis of randomized, controlled trials. Ann Intern Med 1999; 130: 800-9.

17. Quinlan DJ, Mc Quillan A, Eikelboom JW. Low-molecular-weight heparin compared with intravenous unfractionated heparin for treatment of pulmonary embolism. Ann Intern Med 2004; 140: 175-83.

18. Echegaray M, Alonso JL, Urbieta M, Abínzano ML, González C, Solano M. Tromboembolismo pulmonar: evolución a largo plazo y epidemiología clínica. An Med Interna (Madrid) 2003; 20: 451-56.

19. Douketis JD, Foster GA, Crowther MA, Prins MH, Ginsberg JS. Clinical risk factors and timing of recurrent venous thromboembolism during the initial 3 months of anticoagulant therapy. Arch Intern Med 2000; 160: 2341-36.

20. Hansson PO, Sörbo J, Eriksson H. recurrent venous thromboembolism after deep venous thrombosis. Arch Intern Med 2000; 160: 769-74

21. Hull RD, raskob GE, Brant RF, Pineo GF, Valentine KA. Relation between the time to achive the lower limit of the APTT theraputic range and recurrent venous thromboembolism during heparin tratment for deep vein thrombosis. Arch Intern Med 1977; 157: 2562-68.

22. Anand S, Bates S, Ginsberg JS , Levine M, Buller H, Prins M, et al. Recurrent venous thrombosis and heparin therapy. An evaluation of the importance of early activated partial thromboplastin times. Arc Intern Med 1999; 159: 2029-32.

23. Anand S. Ginsberg JS, Kearon C, Gent M, Hirsh J. The relation between the activated partial thromboplastine time response and recurrence in patients with venous thrombosis treated with continuous intravenous heparin. Arch Intern Med 1996; 156: 1677-81.

24. Ridker PM, Goldhaber SZ, Danielson F, Rosenberg Y, Eby CS, Deitcher SR, et al. Long-term low-intensity warfarin therapy for the prevention of recurrent venous thromboembolism. N Engl J Med 2003; 348 1425-34.

25. Schulman S, Wahlander K, Lundstrom T, Classon SB, Eriksson H Thrieve III Investigators. Secondary prevention of venous thromboembolism with the oral direct thrombin inhibitor ximelagatran. N Engl J Med 2003; 349: 1713-21.

26. Kearon C, Ginsberg JS, Kovacs MJ, Anderson DR, Wells P, Julian JA, et al. Comparison of low-intensity warfarin therapy with conventionalintensity warfarin therapy for long-term prevention of recurrent venous thromboembolism. N Engl M Med 2003; 349: 631-9. 